Revue de l'Institut des langues et cultures

d'Europe, Amérique, Afrique, Asie et Australie

$45 \mid 2022$

Femme(s) et sorcellerie en Espagne et en Italie à l'époque moderne

\title{
«Sarà via mai striga? " : sorcières de ville au prisme des conventions comiques sur la scène italienne de la Renaissance
}

"Sarà via mai striga?": Urban Witches through the Lens of Comedic Norms on the Italian Renaissance Stage

\section{Costanza Jori}

\section{OpenEdition \\ Journals}

Édition électronique

URL : https://journals.openedition.org/ilcea/14479

DOI : 10.4000/ilcea.14479

ISSN : 2101-0609

Éditeur

UGA Éditions/Université Grenoble Alpes

\section{Édition imprimée}

ISBN : 978-2-37747-330-4

ISSN : 1639-6073

Référence électronique

Costanza Jori, « "Sarà via mai striga? » : sorcières de ville au prisme des conventions comiques sur la scène italienne de la Renaissance », ILCEA [En ligne], 45 | 2022, mis en ligne le 31 janvier 2022, consulté le 31 janvier 2022. URL : http://journals.openedition.org/ilcea/14479 ; DOI : https://doi.org/ 10.4000/ilcea. 14479

Ce document a été généré automatiquement le 31 janvier 2022.

(c) ILCEA 


\title{
«Sarà via mai striga? » : sorcières de ville au prisme des conventions comiques sur la scène italienne de la Renaissance
}

\author{
"Sarà via mai striga?": Urban Witches through the Lens of Comedic Norms on \\ the Italian Renaissance Stage
}

Costanza Jori

Cette bonne personne possède au bout de la ville,

là-bas, près des tanneries, au bord de la rivière, une maison isolée, à moitié en ruines [...].

Elle avait six métiers : couturière, parfumeuse, fabricante de fards, réparatrice de pucelages, maquerelle et un peu sorcière. (Fernando de Rojas, La Célestine)

« Et una certa monna Maggiorina che racconcia l'ossa per Roma manda i gridi al cielo per esserli stato solo ripportato ch'io l'ho per una strega» (Aretino, 2014:9): dans le prologue de La Cortigiana de 1525, l'Istrione affiche sa crainte de donner le coup d'envoi à la représentation face aux récriminations de personnages issus de la vie réelle romaine, indignés d'y être pris pour cible et de voir leur réputation écornée ${ }^{1}$. Par ce jeu narquois de détournement des codes, Pietro Aretino promeut sa conception d'un théâtre qui se voudrait le reflet documentaire du monde extérieur, lequel fait brutalement irruption au sein des structures de la comédie régulière en train de s'affirmer. Faisant voler en éclats la frontière rassurante qui sépare fiction et réalité, le prologue fait allusion, à côté de deux figures de courtisans et charlatans historiquement identifiables, à une femme désignée précisément par son activité de guérisseuse suspecte de sorcellerie, censée être au même titre que les autres une spectatrice réelle, mécontente et menaçante, de la représentation à venir. Il s'agit là d'une figure à l'identité vague, mais dont le prénom à consonance familière produit un effet de réel : elle semble bien se 
trouver là au même titre que les autres en tant qu'emblème immédiatement reconnaissable, par son genre et la catégorie socio-professionnelle dont elle relève, d'une Rome coda mundi, babélique, corrompue. Et la façon dont Prologo Istrione évoque avec une ironie mordante tout un engrenage de rumeurs et intimidations ( ripportato », « io l'ho per una strega ») n'est peut-être pas sans entrer en résonance avec le contexte particulier des premières vagues de procès de sorcellerie : on sait le poids que les juges accordaient à la réputation au sein de la communauté, à la dénonciation de voisins («l'hai tu per strega? » est une question récurrente lors des interrogatoires de témoins des procès inquisitoriaux) ${ }^{2}$. Maggiorina est par ailleurs le seul personnage supposément "historique» convoqué dans ce dialogue liminaire, tissé d'effets d'annonce trompeurs, à être mentionné au cours de la pièce, fût-ce in absentia, dans la bouche de l'entremetteuse Aloisia. Celle-ci se présente comme sa fervente disciple et paraît justement sur scène en annonçant à son comparse Rosso que sa vieille maîtresse sera brûlée à l'aube du jour suivant, "si abrucia domatina» (Aretino, 2014: 100) - comme si l'auteur mettait en œuvre une sorte de malicieux télescopage métathéâtral de temporalités.

2 Si nous avons insisté, en guise de préambule, sur ce court-circuit entre fiction et réalité qui préside à la version 1525 de La Cortigiana, c'est parce que Monna Maggiorina nous semble constituer la toute première représentante d'une longue lignée de vieilles femmes s'adonnant, entre autres activités, à la sorcellerie dans plusieurs comédies italiennes de la Renaissance; elles s'inscrivent dans le sillage de La Célestine de Fernando de Rojas, bien que leur rôle soit souvent secondaire par rapport à leur modèle espagnol ${ }^{3}$. Après les premières apparitions plus ponctuelles dans la première moitié du siècle, entre Rome et Bologne, avec les créatures d'Aretino et Artemona dans ITre tiranni d'Agostino Ricchi ${ }^{4}$, c'est la production de l'aire nord-orientale dans la période contemporaine et postérieure au concile de Trente qui nous offre la galerie la plus riche. Nous trouvons donc Agata, Cortese et Ortica dans la comédie plurilingue vénitienne des Liquidi, avec Gigio Arthemio Giancarli (La Zingana, Mantoue, 1545), Andrea Calmo (Il Travaglia, Venise, 1546), Marin Negro (La Pace, Venise, 1561) ${ }^{5}$, Donnola dans l'une des trois comédies de Luigi Groto (Il Tesoro) ${ }^{6}$ et Medusa dans Il Fedele, l'unique pièce d'Alvise Pasqualigo ${ }^{7}$. La Strega de Lasca ${ }^{8}$, avec son personnage « éponyme » Mona Sabatina, se rattache au contexte culturel florentin, bien qu'éditée à Venise. La richesse du corpus de l'aire nord-orientale n'est guère étonnante, reflet de la dense présence des streghe/herbere dans la Sérénissime, en ville et dans les zones plus enclavées des vallées alpines marquées par les vagues de bûchers avec leur cortège de conflits et de controverses sur le sabbat; le centre et la Toscane ont connu des phénomènes analogues ${ }^{9}$. Notons au passage l'éclipse de ce type comique dans la production théâtrale napolitaine et méridionale, sans doute du fait que dans ces territoires le phénomène du sabbat et les persécutions ont été moins intenses ; à peine Aloisia fait-elle une allusion ironique au célèbre noyer de Bénévent, où est censée se rendre Maggiorina grâce à son onguent, muée en chat $^{10}$. Dans ces laboratoires d'expérimentation dramaturgique, linguistique et spectaculaire de la comédie dite «régulière » que sont la Rome pontificale des années 1520, puis Florence et surtout Venise dans la deuxième moitié du siècle, se met vite en place une véritable topique du personnage, dont le "portraitrobot" conjugue une série d'attributs physiques, moraux et socio-professionnels récurrents ; à cela s'ajoute un éventail codifié de fonctions au sein d'intrigues nourries de beffe et de tromperies à la chaîne. C'est sur ces schémas invariants que va donc se focaliser notre étude, même si cela risque de donner lieu à d'inévitables 
schématisations par rapport à une catégorie excessivement générique comme la comédie de la Renaissance ${ }^{11}$.

3 Mais si notre réflexion s'est ouverte par le dispositif arétinien, c'est aussi qu'il préfigure la façon dont s'agencent conventions théâtrales, lignages littéraires et vocation documentaire dans des comédies dont le pouvoir de «rispecchiamento » du réel, selon le célèbre précepte comœdia est imitatio vitæe, speculum consuetudinis, constitue la profession de foi de nombreux dramaturges: leur production nait sous le signe d'une tension féconde entre tradition et modernité. Ce type comique et pittoresque se constitue à partir d'un jeu d'hybridation qui décompose et recombine les attributs de la ruffiana issue des comédies antiques et de la novellistica, avec un certain nombre de traits qui reflètent la réalité historique de l'époque; la magie blanche ou noire, naturelle ou cérémonielle, est en effet partie prenante du quotidien de nombreuses couches de la population, du haut en bas de l'échelle sociale. En ce sens, nos sorcières aux prénoms hautement symboliques constituent les doubles féminins des astrologhi et negromanti plus ou moins charlatanesques qu'affectionnent Ariosto, Della Porta ou Bruno. Ces derniers ont retenu davantage l'attention de la critique, sans doute du fait d'une plus grande variété et plasticité par rapport aux conceptions de la magie de la Renaissance ${ }^{12}$.

Quelques précisions terminologiques préalables s'imposent en partant de ce lieu stratégique du paratexte qu'est la liste de dramatis personce. Le critère de l'âge constitue le seul trait définitoire pour Ortica (vecchia veneziana) dans La Pace et pour Sabatina (vecchia, vedova); et c'est par la seule profession de ruffiana que les vieilles Agata, Donnola, Artemona et Cortese (greca ruffiana) sont désignées - seule Medusa étant définie d'emblée comme incantatrice. Cette omission, sans doute marque de prudence liminaire, dessine d'emblée le caractère problématique de la catégorie même de «strega ». Comme nous le verrons, le pivot des comédies est toujours la réputation, le regard et le discours porté sur ces vieilles qui jamais ne s'auto-représentent de la sorte. Ce n'est qu'en croisant les épithètes employées par les autres personnages (du plus neutre «strega/stria/maliarda» à l'apostrophe injurieuse "stregaccia») avec les techniques magico-thérapeutiques qu'elles mettent en œuvre que nous avons pu identifier notre sous-groupe de "maquerelles un peu sorcières", pour paraphraser Rojas. Ce qui permet justement aux interlocuteurs (relais des lecteurs/spectateurs de l'époque) de qualifier les personnages de sorcières, c'est l'exercice d'un certain nombre d'activités dont le périmètre renvoie à la catégorie socio-professionnelle des «streghe/ herbere » qui s'affirme précisément tout au long du Xvi siècle, en particulier dans les villes italiennes ; l'ancrage urbain de nos comédies avec leur « veduta di città » de Rome, Florence, Venise, Trévise ou Adria, s'y prête tout à fait ${ }^{13}$. Ces femmes souvent seules, ou veuves, vieilles ou plus jeunes, parfois proches du milieu des courtisanes, vivent de petits métiers et d'activités à la marge allant du sortilège assorti d'invocations aux démons, à la divination, à des remèdes pour soigner un maléfice - ce qui peut donner lieu à des condamnations allant du pilori au bannissement, jusqu'au bûcher. Dans le cadre du pluralisme thérapeutique propre à la première modernité, les techniques relevant de la médecine empirique, de l'obstétrique à la cosmétologie, participent de cette polyvalence très bien documentée par une abondante production d'études historiques ${ }^{14}$.

5 Or, même si l'imaginaire maléfique et fantastique du sabbat, voire la simple invocation de démons sont prudemment évincés de l'univers quotidien et urbain de la comédie, la transposition théâtrale de tels phénomènes est évidemment loin d'être neutre. Alors 
que la démonologie commence à fixer un certain type de narration autour de la sorcière, ce dont témoignent la diffusion du Malleus maleficarum édité en 1486 ou du dialogue de Pico della Mirandola qui en reprend certains thèmes, la mise en place de l'arsenal répressif suscite débats et controverses. Les conceptions intransigeantes de théologiens et inquisiteurs se heurtent à un front plus sceptique, composé de juristes, philosophes ou médecins. Les objections portent sur la réalité physique du sabbat, sur la matérialité du corps des démons, la compatibilité entre providence et diffusion d'une secte démoniaque, mais aussi sur la fiabilité des dénonciations des complices sous l'effet de la torture; à partir de la première moitié $\mathrm{du} \mathrm{XVI}^{\mathrm{e}}$ siècle, avec des figures comme le médecin Girolamo Cardano (1501-1576) puis Giovan Battista della Porta (1535-1615), commence à s'élaborer une interprétation naturaliste de la sorcellerie ${ }^{15}$. Ainsi, avec ce champ de discours en toile de fond, nous tâcherons de mettre au jour la façon dont la représentation, dans sa stéréotypie même, semble condenser et relayer une série de topoï négatifs hérités d'une tradition misogyne ayant conflué en partie dans les manuels de démonologie, faisant de nos sorcières un anti-modèle, des figuresrepoussoir grotesques du désordre et de la corruption morale, dont les techniques et les savoirs magico-thérapeutiques semblent discrédités. En un deuxième temps, par la mise en perspective de l'imagerie charlatanesque, il s'agira de mettre aujour l'ambivalence et les paradoxes qui sous-tendent la représentation. Les valeurs, principes de comportement, savoirs qui leur sont associés se découvrent sous un jour bien plus complexe - le négatif, dans une inversion de signes, pouvant se charger d'une virtualité positive. Et dans l'émergence de cet espace de liberté et d'autonomie accordé à des personnages féminins affranchis d'un certain nombre de figures d'autorité, se donne peut-être aussi à lire en filigrane le processus de "disincanto delle arti diaboliche » mis en lumière par Matteo Duni (2012 : 205), qui a lieu dans la péninsule entre la fin du XVI ${ }^{\mathrm{e}}$ et le XVII ${ }^{\mathrm{e}}$ siècles.

Les portraits physiques de nos sorcières, morceaux de bravoure rhétorique confiés à un serviteur ou à un parasite, sont tributaires d'une tradition littéraire misogyne remontant au Moyen Âge, où la laideur repoussante est à l'image de la flétrissure morale. La décrépitude physique donne lieu à de savoureux contre-blasons, avec parfois une allusion ironique à la balafre au visage, peut-être marque diabolique comme pour Célestine. Il suffit de lire le portrait de Donnola dressé par le valet Cornacchia au jeune Girofilo amoureux de Licinia, un potentiel client :

Presso la casa di messer Zelotipo / Marito de la vostra Licinia, habita / Una vecchia barbuta,

a la qual lagrima / Sempre un occhio, [...] / ha il viso attraversato d'un notabile / Freggio, $e$

la ciglia irsute si congiungono / [...] e se sta il giorno in chiesa prattica / Tutta la notte poi ne cimiterij, / o tra le forche, dove i rei s'appiccano, / [...] / Sa movere / Con le parole sue sole a lussuria. (Groto, 1599 : 10-11)

Sous le signe de la cupidité et de la duplicité bigote, Groto désigne un savoir (le verbe sapere en anaphore) qui fait le lien entre les métiers de maquerelle et d'ensorceleuse aux pratiques nécrophiles: selon le paradigme célestinesque, l'art de la magie ad amorem vient doubler et relayer les techniques de la rhétorique persuasive, surtout dans le but de contrer les réticences de jeunes filles ou d'épouses tenues par les codes de l'honneur et de la réputation. C'est ce que souligne Medusa dans Il Fedele, le but commun étant de " convertire la volontà della persona amata in maniera, che quel che voleva l'amante vuol essa anchora ». C'est bien cela qui la rend identifiable comme "strega ", comme en témoigne cet échange anodin au détour d'une rue entre deux servantes: « PANFILA: Dove vai? BEATRICE: A trovar una strega per la mia patrona, che spasima per amor del 
signor Fortunio. Panfila: E che vuole da lei? Beatrice: Che faccia malie, che lo sforzino ad amarla. » (Pasqualigo, $1576: 22$ )

7 La panoplie de recettes convoquées sur scène est parfaitement représentative des pratiques de l'époque: figurines en cire, philtres et galettes concoctés à partir de plantes et fragments organiques, ongles ou os broyés de cadavres humains, qu'il faut se procurer dans le plus grand secret; techniques divinatoires comme le fait de " gettare la cera » et " gettare le fave », répandues notamment parmi les courtisanes vénitiennes ${ }^{16}$; enfin, emprunts à la liturgie catholique avec par exemple, dans l'aire vénitienne, la circulation clandestine des orazioni de l'Agnolo bianco ou l'utilisation d'eau bénite ou de terre consacrée de cimetières. Néanmoins, si l'on s'attache à définir la fonction attribuée à ces «stregherie » au sein de l'univers comique, on est frappé d'entrée de jeu par le hiatus entre l'espace verbal qui leur est assigné dans le discours et le rôle relativement marginal qu'elles jouent dans la fabula elle-même, en tant que facteur ayant le pouvoir effectif de modifier la réalité, ici le domaine des affects des personnages. De façon symétrique, la scène de magie/sorcellerie, avec sa charge de séduction spectaculaire liée à l'emploi d'accessoires et de formules incantatoires (fût-elle parodique, et l'on songe, pour ce qui est des faux negromanti, à l'attirail d'astrolabes, de cercles, et de barbes postiches), tend à être prudemment reléguée dans l'espace du hors-scène. L'écart par rapport au paradigme célestinesque est sensible: après avoir promis ses services au noble Calixte via son serviteur Sempronio, la vieille maquerelle, seule en scène, conjure Pluton et les démons tout en opérant une manipulation sur un fil imprégné de venin qu'elle ira vendre à Mélibée. Cette dernière se convertit alors brutalement à l'amour pour Calixte qui aura les conséquences fatales que l'on sait - un revirement qui a suscité des divergences d'interprétation, entre la reconnaissance d'une action réelle de la magie sympathique et du pacte démoniaque et une lecture plus naturaliste et sceptique, à la lumière de la puissante maïeutique persuasive de la vieille, à même de révéler la jeune fille à son être le plus profond ${ }^{17}$. La caractérisation caricaturale d'Aloisia comme sorcière s'en inspire directement, dans un jeu d'échos intertextuels, ce qui donne lieu à de savoureux échanges avec son comparse Rosso : évoquant sous forme d'antiphrases sarcastiques les sombres méfaits dont est accusée sa maîtresse sorcière, celle-ci déploie dans une longue tirade pittoresque la liste d'ustensiles et d'ingrédients reçus en héritage. Le topos burlesque du testament sous forme de liste, avec ses parallélismes, paronomases et séries ternaires, les détails raillant avec un humour macabre des pratiques à la limites de la nécrophilie, l'apparentent à l'étalage verbal d'une tirade de bonimenteur de foire : «[...] un fiasco de lacrime d'amanti, un bichiere di sangue di nottola, ossa di morti per tormenti et per tradimento, unghie de gufi, cuori d'avoltori, denti di lupi, graso d'orso e funi di impicato a torto. " (Aretino, 2014 : 102) Pourtant cet attirail n'est appelé à jouer aucun rôle par la suite, comme si Aretino créait une fausse piste et soulignait à dessein le principe de l'accessoire, du désordre contingent qui régit l'univers comique avec ses beffe arbitraires et éphémères, sans lien aucun avec un projet construit, avec la puissance d'un désir amoureux. La magie vraie ou fausse n'a même pas lieu d'être, Aloisia se limitera à remplacer la dame convoitée de Parabolano par la fornaia, dans une relecture dégradée et totalement immanente de la source espagnole. Dans Il Fedele, Medusa se rend sur le sagrato de l'église en compagnie de sa cliente, la gentildonna Vittoria, dont le mari est absent et qui, tyrannisée par une passion qui la rend cruelle et insensible à son premier amant Fedele, est prête à lui donner beaucoup d'argent pour se faire aimer du misogyne Fortunio. Il s'agit là de l'unique scène du corpus où un rituel magique interdit est mimé 
directement sur scène, dans une atmosphère occulte et nocturne. Elle s'y adonne à une véritable promotion publicitaire de ses recettes sur le registre prescriptif des manuels de secrets, sortant à chaque fois de sa besace une plume de corbeau ou une fiole de lait maternel pour concocter une galette; puis elle officie avec la figurine de cire choisie par sa cliente : «Bisogna scaldarla, pungerla, e costringere questi spiriti scritti qui sopra, e poi sarà fatto. [...] Astarot, Buffon [...] Vi scongiuro ministri di Satanasso, per le virtù tremende di Amodeo [...] ch'andiate a ritrovar in quest'hora Fortunio. " (Pasqualigo, 1576:42) Cette exhibition rituelle avec utilisation symbolique et performative de la parole proférée et écrite apparente Medusa à une actrice tenant son auditoire et semble directement tirée des actes de procès pour "stregheria $»^{18}$. Elle la rend d'ailleurs immédiatement identifiable par le serviteur qui les espionne en cachette : « NARCISo: In Sacrato? Al corpo della barba mia, che queste sono streghe. ( (Ibid. : 39) Néanmoins le hasard - encore un jeu de fausses pistes avec les attentes du spectateur - fait que Medusa, interrompue par le pédant Onofrio, s'enfuit à toutes jambes du cimetière avant d'en avoir terminé, si bien qu'il faudra qu'elle fasse appel non plus à la magie mais à son art de la manigance pour parvenir à retrouver une maîtrise sur l'évolution du chassé-croisé amoureux. La chute comique dépossède donc une fois de plus la «stregheria ", dont on ne sait si elle aurait été efficace ou pas, de sa fonction dramatique effective - mais la dimension sulfureuse, cupide et charlatanesque de Medusa n'en est pas moins affirmée, en dépit de cet escamotage.

8 Les autres comédies du corpus nous offrent une perspective aussi prudemment « désenchantée ». Les compétences de sorcières de Donnola, Agata ou Cortese ne rentrent en jeu la plupart du temps qu'au niveau des beffe secondaires, ourdies aux dépens des barbons libidineux, pères, mères ou maris qu'il s'agit de duper, par exemple pour les éloigner de la maison où doivent se rencontrer en secret les jeunes premiers. Voici les instructions d'Agata à sa vieille cliente qui convoite le jeune Cassandro, pour qu'elle sorte se procurer clandestinement les ingrédients : «In prima besogna che vu andé a tuor con le vostre man l'acqua de sette pille d'acqua santa, [...] e della terra ch'abbia coverto sette morti; e lassé puo far a mi, che se [...] el cuor de m. Cassandro fosse pì duro che un baston, el farò pì umele ca la cera.» (Giancarli, 1991: 393) L'on sera sensible ici à l'habile promotion «publicitaire» en dialecte de sa propre expertise magique, exploitant la symbolique des nombres et l'antithèse entre les deux comparatio de la dureté du bâton et de la cire malléable, typiques des formules incantatoires fondées justement sur un principe d'analogie sympathique. Il est néanmoins évident qu'ici l'opératrice ne croit nullement que cela agira sur le jeune homme, même si les ingrédients n'ont rien de fantaisiste par rapport à l'époque. Dans La Strega de Grazzini, Mona Sabatina se borne à cacher dans sa maison deux amants clandestins, Orazio, revenu à Florence sous une fausse identité et la jeune Violante, en sa qualité de ruffiana. Le rôle moteur de l'intrigue est en quelque sorte confisqué par Fabrizio, ami d'Orazio, qui tire parti de sa réputation de sorcière pour ourdir ses manigances :

Sapendo che io sono amicissimo di Monna Sabbatina, la quale pensa che sia qualche gran donna nello stregare e nelle malíe, mi narró l'amore di Taddeo suo nipote [...]. Io subito gli dico che non fu mai negli incantesimi maggiore donna da Circe in qua.

9 Il fait croire au père d'Orazio, le marchand Luc'Antonio, plutôt sceptique envers cette «stregaccia », que Sabatina a eu la révélation « per via di diavoli » du retour de son fils en ville, ce à quoi son comparse Neri s'exclame: "Odi, ella ha anche nome di strega!» (Grazzini, 1976: 62-63) En parallèle, il fait croire à deux vieux, Bonifazio et Bartolommea, que Sabatina va concocter un sort afin que Geva, fille de Luc'Antonio, 
tombe amoureuse de leur fils et neveu Taddeo. Celui-ci, par dépit amoureux, veut partir à la guerre, risquant de mourir et de les priver de la jouissance de son patrimoine. La cupidité et la crédulité sont la clé de voûte, à tous les niveaux, de l'intrigue : Fabrizio, simulant une prescription de Sabatina, demande de l'or pour fabriquer la figurine servant au sortilège, topos des pièces fondées sur la fausse magie. Le titre n'est donc pas sans induire quelques effets d'attente ironiquement déjoués, puisque "la strega " se réfère à la réputation sociale de Sabatina, le personnage éponyme se voyant finalement comme dépossédé de son "protagonisme" et même de sa potentielle identité charlatanesque par Fabrizio, jusqu'au dévoilement final assorti de mariage réparateur qui adopte une perspective démystifiante : «FABRIZIO: [...] vi diceva ch'egli era vivo perch'io lo vedeva ogni ora, e non perché la vecchia me lo rivelasse come strega o maliarda, che son tutte quante baie e ciance. » (Ibid. : 106)

10 Ainsi, ces vieilles cupides qui favorisent par leurs manigances et sortilèges, vrais ou faux soient-ils, une forme d'amour transgressif hors mariage et/ou adultérin, semblent se dresser comme les icônes négatives et grimaçantes d'un désordre qui sape allègrement les fondements d'un ordre social centré sur l'institution matrimoniale et le culte de la virginité, en particulier pour ce qui concerne la production vénitienne, postérieure aux directives du concile de Trente. Le retour à l'ordre final sanctionné par les mariages n'atténue qu'en partie la dimension transgressive de l'éros qui anime les intrigues : le fait que ces vieilles femmes ne soient jamais démasquées ou châtiées pour leur double activité de maquerelles/ensorceleuses par une quelconque instance institutionnelle participe de ce triomphe de valeurs non conformes à la morale chrétienne. L'association indirecte entre vieillesse et luxure, $d u$ fait que nos envoûteuses, parfois ex-courtisanes encore bonnes vivantes, font miroiter auprès de leurs clients jeunes ou vieux une consommation charnelle non directement vouée au mariage ni à la reproduction n'est peut-être pas sans faire écho, en filigrane, à l'imagerie du sabbat chère aux inquisiteurs: la vieille strix stérile et débauchée campée par Pico della Mirandola en est l'emblème répulsif. Cette négativité morale semble aller de pair avec une entreprise de rabaissement des «stregherie » à une forme d'imposture charlatanesque ou d'ignorance superstitieuse, discréditant autant les maitres trop crédules que les opératrices elles-mêmes ; une négativité qui semble donc se détacher sur ce point de l'imaginaire inquisitorial, puisque nier la réalité de ces pouvoirs démoniaques, même si cela relève de conventions comiques, pouvait ne pas être au goût des inquisiteurs qui menaient la plupart des procès sur ces chefs d'accusation.

11 Néanmoins, la récurrence de ces constructions en trompe-l'œil, avec des sortilèges dont on ne peut guère vérifier l'efficacité du fait de leur inachèvement, peut se donner à lire dans une perspective différente, presque inversée, comme facteur d'ambivalence. Une comparaison avec les faux negromanti ou astrologhi des comédies d'Ariosto ou Della Porta est parlante. Ceux-ci théorisent verbalement leur imposture, ce sont des charlatans itinérants qui assument une fausse identité - une perspective qui peut être interprétée dans le cas du philosophe napolitain comme une stratégie défensive, son intérêt pour la magie naturelle et l'astrologie judiciaire lui ayant valu d'être accusé de nécromancie par le Saint-Office ${ }^{19}$. Or, nos comédies n'accueillent guère de monologue ou d'aparté autoréflexif qui dévoilerait une position de duplicité tranchée des opératrices face à l'ensemble des procédés magico-thérapeutiques qu'elles exécutent : quelque chose à la marge semble échapper de ces créatures dont le large éventail de 
pratiques rend problématique toute délimitation tranchée entre expertise et imposture. Mais on peut y voir aussi l'indice d'un regard plus nuancé vis-à-vis de ces compétences. Et si l'on prend en compte, en élargissant la focale, la façon dont les dramaturges caractérisent ces personnages au-delà des sortilèges directement opérants dans la trame sentimentale, par le biais des portraits et descriptions qui émaillent le discours des uns et des autres, alors la ligne de partage entre vrai et faux savoir, feinte et sincérité, valeurs et savoirs positifs et négatifs, se découvre bien plus poreuse et mouvante.

Les comédies du corpus mettent en scène une polyvalence qui permet à des personnages dans la nécessité, femmes seules, veuves, ou dotées d'un mari fainéant, de subvenir à leurs besoins, s'affranchissant de la tutelle masculine; elle peut aussi leur conférer une forme d'humanisation, notamment dans les pièces dialectales à Venise. Dans La Zingana de Giancarli, Agata compte de nombreux monologues en dialecte où elle s'adresse au public, affairée dans ses micro-activités : cela lui permet de bien élever sa fille et de s'affranchir de la domination d'un mari ivrogne, mais aussi, clin d'œil méta-théâtral jouissif, de s'offrir quelques menus plaisirs comme assister à un spectacle comique de Burchiella. Elle paraît dans la rue au petit matin, apostrophant le public féminin car, s'apprêtant à faire sa tournée dans le voisinage, elle a confondu deux ustensiles, la petite casserole pour faire son sortilège divinatoire et celle servant à faire fondre cette même cire pour épiler ses clientes :

«Ma donde hogio il cervello, grama mi? No hogio tolto la pignatella dal sguàrdolo in scambio per quella de buttar la cera? Esì aveva mo impromesso a madonna Viena d'andar stamattina a dezun da essa a butarghe la cera e dirghe la 'razion de l'Anzolo bianco...» (Giancarli, 1996 : 209)

Le micro-gag du "pignatello", un objet par ailleurs très présent dans les archives de procès vénitiens, matérialise avec une grande efficacité scénique la superposition entre l'activité d'esthéticienne et celle d'ensorceleuse : il ancre la magie dans une dimension artisanale, concrète et prosaïque de petit métier utile à la communauté, dans une menue sociabilité féminine également, avec la déclamation des oraisons illicites pour les voisines et comari. Un peu plus loin, avec un langage cru et direct, le vieil Acario l'identifie comme la guérisseuse barbue "chié cava li vermi del culo a $i$ fandulini» (Giancarli, 1991: 227) - peut-être un pied de nez ironique à l'imagerie maléfique et surnaturelle de la sorcière dévoreuse d'enfants. Dans un autre registre, Aloisia, ironiquement qualifiée par Rosso de «specciala ", hérite outils et compétences empiriques utiles à la clientèle du quartier héritée de Maggiorina: «[...] per sua grazia son sempre la prima chiamata a nettare denti, a cavar la puzza del fiato e mille gintilezze.» (Aretino 2014:102) Quelques répliques à la marge traduisent la reconnaissance d'une expertise, typiquement féminine, dans le domaine de l'obstétrique/gynécologie : la sorcière représente une figure ambiguë de sage-femme/ avorteuse, sans oublier son savoir-faire de raboteuse de virginités, un topos de la littérature érotique s'inspirant de recettes et de subterfuges recensés dans les nombreux livres de secrets de l'époque. Dans la version vénitienne de 1534, Aloisia a reçu en héritage "il lattovaro da impregnare e da spregnare» (ibid.:568); Agata apostrophe le public féminin en sollicitant quelques langes pour une pauvre jeune fille qui vient d'accoucher chez elle après avoir été abusée par son amant.

Les conventions scénographiques de la comédie régulière illustrent l'inscription de ces personnages féminins dans une dynamique d'échanges dont elles sont les agents actifs. La maison de l'entremetteuse, lieu canonique de la scène comique de la Renaissance 
selon la théorisation de Serlio, doit jouxter, sur la place publique, l'église et la taverne : devenue accessoirement antre/laboratoire, elle subvertit l'association traditionnelle au théâtre entre le féminin et la sphère intime, privée, et domestique. Dans le hors-scène soustrait au regard du public, derrière la façade des praticables, se déploient les multiples activités professionnelles illicites ou semi-licites menées par ces vieilles femmes et leurs servantes, de la magie à la divination à la prostitution. Cette maison constitue le pivot de tout un réseau de micro-échanges et transactions verbales, érotiques et économiques. Les maîtres, de respectables cittadini ou plus souvent leurs serviteurs, viennent toquer à la porte ou héler les servantes à la fenêtre pour demander des services, comme le marchand Luc'Antonio dans La Strega qui habite en face, Beatrice la servante de Vittoria dans Il Fedele; ils craignent parfois qu'on ne les aperçoive sur le seuil de ce lieu malfamé, tel le valet Cornacchia qui fustige la tendance à la délation des habitants d'Adria. Sous le signe d'une alliance paradoxale entre le secret et la "publicité », la centralité et la marge, ces praticiennes sont donc autant sédentaires (ce qui les distingue des faux magiciens itinérants ariostesques, étrangers à la ville) que mues par un mouvement frénétique et centrifuge vers l'extérieur, l'univers plus large de la cité. Dans la description d'Aloisia, l'effet-liste montre que Maggiorina maîtrise l'espace urbain, social et langagier, inversant les hiérarchies de genre: "Al beccaio, al pizzicagnolo, al mercato, a la fiera, al fiume, [...] al barbiero, alla gabella, a la taverna con cuochi, mesi, preti, frati e fra soldati, sempre sempre toccava favellare a lei, era una Salamona tenuta. » (Ibid. : 105) Ailleurs, on voit nos vieilles traverser la place, toquer aux portes, sans oublier, bigoterie oblige, la fréquentation des églises; de savoureux récits les dépeignent tandis qu'elles pénètrent dans l'espace invisible du hors-scène, pour corrompre une jeune fille, épiler une voisine, acheter chez l'apothicaire poudres et plantes, voire aider à un avortement clandestin. On les imagine s'aventurer jusqu'aux lieux plus solitaires et inquiétants, les cimetières, la montagne pour cueillir des herbes à la tombée de la nuit - Fileno dit qu'Artemona était absente de chez elle, « andata fuori al monte / a cercar di certe erbe " (Ricchi, 1998: 34). Cette capacité de médiation interclasse, leur autonomie socio-professionnelle et leur âge leur confèrent la liberté de traverser les espaces, de franchir le seuil entre le privé et le public, le profane et le sacré, le familier et l'étranger, la ville et la campagne. Elles se distinguent ainsi de la condition des jeunes premières recluses dans l'espace domestique, soumises à l'autorité d'un mari ou d'un père, ce dont se plaint par exemple la jeune Licinia, mariée contre son gré et qui va recourir aux services de Donnola, face à sa mère Prudenzia dans Il Tesoro ${ }^{20}$ de Groto.

Dans I Tre tiranni, Fileno consacre une très longue hypotypose à la descriptio de la maison d'Artemona, où il s'est rendu pour solliciter ses services. S'il enchaîne au début les topoï misogynes, évoquant des "vecchie sciancate che paion Creonte » concoctant dans une immense marmite un "belletto " répugnant à base de crânes de chauve-souris, la "casaccia antica" de cette communauté de vieilles sorcières, où logent aussi quelques filles vouées à des activités équivoques, se mue à travers son regard émerveillé en laboratoire d'alchimiste ou d'apothicaire. La description visionnaire s'attarde sur une liste de détails pittoresques avec une précision terminologique savante: «[...] lambicchi e campane da stillare, / bocce di vetro le più contrafatte / del mondo. Ivi fornaci, scaffe e stufe / [...] / Per le fenestre fiori, erbe e sementi, / radici, zucche, zucchelle e pignatte [...]. » (Ibid. : 35-36) Cet antre/laboratoire est singulièrement représenté comme un lieu d'initiation voué au savoir et à un partage du travail exclusivement féminin, une étonnante microsociété qui sous ses atours burlesques repose sur une organisation rationalisée et 
collective du travail, un partage des tâches horizontal : «Ivi tutte hanno / il lor proprio esercizio: una pesta ossa / [...] un'altra l'herbe / mette ne le strettoie e cava il sugo; / questa fa medicine; un'altra unguenti [...]. » (Ibid. : 35)

16 Ces éléments contribuent à faire de nos sorcières les dépositaires paradoxales d'une autorité bien différente de celles qui repose sur les livres dont se réclament les figures de pédants qui hantent la scène dans la deuxième moitié du siècle. Notons que l'observation et la connaissance empirique du fonctionnement élémentaire du corps humain et de la nature (les propriétés des herbes à employer pour les sortilèges), s'enrichit de celle des ressorts du désir amoureux (Aloisia décrypte les sentiments de Parabolano à l'inclination de sa tête, avec une compétence physiognomonique), ainsi que plus largement des rapports de domination et des faux-semblants régissant la vie sociale. C'est à ces voix subalternes et à la marge en effet, par le biais de monologues et d'apartés, que les dramaturges confient la mission d'énoncer un jugement critique sur l'univers fait de dissimulation et de passions, d'intérêts et de convoitises égoïstes qui caractérisent maîtres, serviteurs, courtisans, pédants et parasites, et dont elles sont partie prenante. La bigote Aloisia stigmatise l'hypocrisie religieuse et la corruption de la cour romaine, Medusa disserte doctement sur les faiblesses des hommes, en particulier la tyrannie des maris; Agata tourne en dérision la vanité de ses clientes qui se font épiler et lisser la peau. C'est ce qui explique les références à une forme de sagesse et d'autorité associées à la vieillesse qui se charge alors d'une valeur positive, fût-ce avec une nuance ironique - voir Aloisia qui parle de sa maîtresse sorcière Maggiorina comme d'une « Salamona ».

Dans la tragi-comédie de Rojas, l'autorité reconnue à la vieille Célestine lui permet d'instruire Parmenio et Mélibée par une forme de maïeutique certes distincte de ses savoirs magiques de sorcière stricto sensu, mais elle est aussi la " tutrice » en sortilèges et maquerellage de la jeune Elicia $^{21}$. Calquant ce paradigme initiatique, nos comédies mettent en scène des espaces d'apprentissage alternatifs exclusivement féminins, sortes de lignées matrilinéaires de sorcières/maquerelles, bien qu'avec une profondeur bien moindre. Cette dimension de la transmission de savoirs magiques et «secrets " fondés non sur le livre et l'écrit mais sur l'expérience et l'oralité, entre femmes souvent semi-analphabètes, a été par ailleurs très étudiée par l'historiographie sur les sorcières et traverse les témoignages des actes de procès ${ }^{22}$. L'hommage reconnaissant d'Aloisia à l'héritage matériel et symbolique de sa maîtresse sorcière, qui va des savoirs « machiavéliques » aux outils du métier à la clientèle du quartier, fût-ce sous le signe de la subversion ironique des valeurs d'honnêteté et de justice, pourrait se lire comme le miroir inversé de l'initiation à la courtisanerie du benêt Maco par son maître pédagogue Mastro Andrea: cette beffa cruelle et parodique n'accouche d'aucune métamorphose chez l'initié, qui reste prisonnier de ses leurres. Les Ragionamenti du même auteur, publiés à Venise, offrent d'ailleurs un pendant intéressant à la comédie. Dans le Dialogo nel quale la Nanna insegna a la Pippa, la Comare instruit la Balia sur les formules et rituels de sorcellerie aptes à berner les amants crédules, qui sont loin d'être tous issus du menu peuple superstitieux. Les détails techniques émaillant ses tirades didactiques et prescriptives semblent avoir été recueillis par Aretino dans la cité lagunaire avec une curiosité amusée, presque « ethnographique » avant la lettre : on y apprend comment jeter des sorts avec les fèves ou la cire, à déclamer l'oraison de l'agnolo bianco. Ces «stregherie» sont présentées avec cynisme par la Comare comme la clé de voûte d'un univers de simulation et de supercheries. Mais la dimension directe, empirique et pragmatique de ces préceptes, fondés sur une connaissance impitoyable 
des ressorts de l'âme humaine, les distingue des mensonges et mystifications des courtisans. Dans leur logique d'inversion parodique des conventions des traités de comportement destinés aux jeunes femmes de qualité, les Ragionamenti, manuels d'éducation érotique et morale des prostituées et entremetteuses qui s'avèrent aussi " un peu sorcières ", véhiculent une satire virulente de la vie à la cour. Ils miment et subvertissent les modalités des relations d'apprentissage telles qu'elles ont été codifiées dans toute une production littéraire qui en donne une image on ne peut plus idéalisée et illusoire ${ }^{23}$.

Ce type de dispositif est décliné avec d'autres enjeux dans Il Fedele : Beatrice, la servante de la tyrannique gentildonna Vittoria, subjuguée par les rituels magiques exhibés par Medusa au cimetière, instruite par elle sur ses recettes et sur les vices des hommes et des maris, finit par se convertir. Elle proclame vouloir quitter son état subalterne pour aller vivre dans la maison commune et devenir sa disciple dans tous les arts illicites. Cette relation d'apprentissage/filiation s'oppose en un sens à celle, qui échoue et se grippe, entre le noble Fedele, amoureux véhément éconduit par Vittoria, et le pédant Onofrio. Ce dernier tâche en vain de l'amener à gouverner ses affects par des formules en latin creuses, des références érudites déformées et tautologiques qui masquent en fait son propre désir dissimulé pour Vittoria. La dimension initiatique de l'apprentissage de la sorcellerie, avec sa charge désacralisante, parodique et satirique, participe donc de la définition même des personnages. C'est ce dont témoigne le long portrait de Donnola fait par Cornacchia par lequel nous avons commencé, où l'on voit s'opérer un glissement explicite des compétences magico-thérapeutiques à celles pédagogiques : «[...] ha prattica / Poi, quanta haver si può / ne gli incantesimi, / [...] / Aiuta a partorir le donne gravide [...] / Insegna a leggere / A ricamar, e cucire, e trapungere / (e ancor qualche mestier più dilettevole) / A le fanciulle. » (Groto, 1599 : 11)

Ce portrait synthétise ce qui est apparu tout au long de notre analyse, dans la mesure où l'allusion topique à l'initiation à la prostitution, emblème de relations fondées sur l'utile, coexiste avec un enseignement plus désintéressé et potentiellement émancipateur lié à l'apprentissage de la lecture : une façon de brouiller et de saborder ironiquement les hiérarchies entre disciplines et savoirs. La figure rhétorique de l'éloge paradoxal semble tout à fait adaptée pour caractériser le regard porté par les dramaturges sur les vieilles femmes : la coexistence du dénigrement et d'une forme de reconnaissance, la valorisation de comportements et savoirs à la marge de la licéité qui font habituellement l'objet de condamnation sociale. Mais l'intérêt de ces comédies est justement de montrer que ces sorcières existent avant tout en tant qu'objet des discours des autres personnages, dans des dispositifs polyphoniques où les points de vue s'entrechoquent et se relativisent mutuellement, un peu comme la comédie plurilingue vénitienne sape joyeusement les hiérarchies entre toscan littéraire, dialecte vénitien, greghesco, bergamasque. Ces lignes de tension travaillent parfois de l'intérieur le discours d'un même personnage, comme celui des serviteurs/parasites souvent complices, reflet d'un imaginaire social mouvant: elles questionnent bien entendu la vision des dramaturges eux-mêmes. Nous touchons ici aux limites de notre étude, que nous avons choisi d'axer sur les invariants et la stéréotypie. On peut certes inférer de nos analyses que le regard porté sur la sorcellerie n'est pas univoque, ni réductible à une forme de mépris de lettrés envers des superstitions populaires, ou à la volonté de représentants d'un savoir masculin plus institutionnel de dénigrer et de mettre en doute la validité de "secrets » féminins dépourvus d'assise théorique selon une grille de lecture " genrée ${ }^{24}$. Il conviendrait néanmoins de mettre au jour, de façon 
plus spécifique, les conceptions et positionnements propres à chaque dramaturge et le champ socio-culturel dans lequel il s'inscrit. Les œuvres gagneraient ainsi à être replacées dans le cadre du pluralisme thérapeutique de la première modernité ; les conflits entre la masse de praticiens empiriques et les collèges de médecins fisici (dont Ricchi est un représentant), ces derniers s'efforçant par différents statuts de défendre leurs prérogatives, s'expliquent en partie par le fait que les principes et les propriétés d'un certain nombre de remèdes étaient communs à la médecine savante et à la magie blanche populaire, les propriétés des simples, ou les phénomènes d'analogie sympathique. La floraison de livres de recettes et de secrets, qui circulaient d'ailleurs dans différents milieux et à la cour, témoignent de cette contiguïté. Il serait utile de s'interroger sur l'intérêt d'Aretino pour ce filon récemment mis en lumière par la critique $^{25}$, ou la proximité du théâtre des Liquidi avec l'univers de la foire, des bouffons, cantimbanchi vénitiens, sans oublier le rapport, selon le territoire et le contexte, avec le Saint-Office et les instances de la censure, étudié par Plaisance pour le manuscrit de La Strega et ses éditions.

Si l'on est tout de même tenté d'esquisser l'hypothèse que se dégage de l'ensemble de nos comédies l'impression d'un certain scepticisme, c'est dans la mesure où elles semblent questionner la légitimité même de l'emploi de la catégorie de la sorcière, qui en vient presque à être déconstruite par ce jeu de relativisation ironique des discours. L'accent mis sur la réputation sociale, le hiatus entre la prolifération d'images évoquant les pouvoirs plus ou moins maléfiques de ces vieilles - qui pour leur part ne le reprennent jamais à leur compte - et les comportements donnés à voir sur scène semblent presque les muer en écrans de projection des peurs, fantasmes et désirs des membres de la communauté. Le fait que celles-ci ne sont pas définies comme "streghe " dans le corpus para-textuel, l'un des espaces où s'affirme directement la voix de l'auteur de théâtre, suggère que c'est cette dénomination même, avec sa relativité et ses contours fluctuants et instables, qui se trouve mise en perspective et en question par cette réticence à en faire usage. Les procédés mis en lumière semblent bien suggérer que si cette catégorie est problématique, c'est dans la mesure où elle est façonnée et légitimée par les institutions inquisitoriales à l'origine des procès et par le discours théologico-juridique sur lequel elles s'appuient : leur rapport à la réalité - du sabbat notamment dont elles prétendent donner un clé d'interprétation, tout en créant un type de narration qui influence à son tour, par un processus d'intériorisation, les mentalités et les comportements sociaux - est fortement sujet à caution. Nos comédies se dessinent donc comme une sorte d'antidote, de contrepoint voire de joyeux pied de nez au dispositif mis en place par exemple par Pico dans son traité dialogué Strix. Apistio (l'incrédule), l'humaniste sceptique qui assiste au procès de la vieille sorcière finit par être convaincu de la réalité du sabbat démoniaque et des crimes rituels par la confession détaillée de la prévenue elle-même, et par le renfort d'autorités classiques et scolastiques égrenées par ses doctes interlocuteurs, le juge Dicaste et le sage Fronimo. Loin de toute relativisation ludique et ironique, le dialogue nous amène d'une pseudoconfrontation de points de vue à une vision univoque et fantasmée de la sorcière débauchée et vampiresque, ne laissant guère de marge au doute :

STREGA: Toglievamo i bambini [...] gli foravamo sotto l'ugnine con l'ago e ponendovi le labbra a succhiare, ci empievamo la bocca di sangue [...] e parte se ne votava in un bossolo, per fare l'unguento da ungersi le natiche prima che andassimo al gioco. [...] APISTO: Chi v'ha insegnato i rimedi? STREGA: I demoni ${ }^{26}$. 
Nous aimerions conclure par un exemple qui illustre avec beaucoup de grâce l'esprit bien différent qui anime nos comédies. Dans La Zingana, Giancarli campe un tout jeune serviteur fort ingénu qui, à chaque fois qu'il doit interagir avec Agata pour le compte de son maître, exprime par des exclamations très spontanées une sorte de terreur archaïque enfantine, convoquant l'imagerie la plus maléfique du sabbat et des suceuses de sang d'enfants colportée par Pico ou le Malleus : « FIORETTO: Quella vecchia vecchia, che mangia i bambini..., sapete con quel bastone, e con la barba... [...]. Pur ch'ella non venga poi stanotte, quando io dormirò, a forarme la panza... [...]. FALISco: Ah, ah, ah!» (Giancarli, 1991 : 237, 241). Le contraste avec la dimension prosaïque des activités magiques exercées par Agata en scène suscite le rire non seulement de ses interlocuteurs, mais du spectateur lui-même. La question semi-rhétorique qu'elle pose, affichant son incrédulité lorsque son comparse en proie au doute lui demande au petit matin si elle ne s'est pas rendue au sabbat "con il collegio di Valcamonica", parce qu'elle se frotte les yeux de fatigue, se charge peut-être d'une portée méta-théâtrale : "AGaTA: Mo che distu? [...] Sarà via mai striga? FALISCO: Che so io... » (Ibid. : 221). Agata semble ici s'interroger non seulement sur son identité sociale, mais aussi sur celle de son personnage-type de théatre: faisant vaciller les certitudes, suspendant le jugement de son interlocuteur, relais du lecteur/ spectateur, elle questionne ainsi un imaginaire collectif et littéraire beaucoup plus large $e^{27}$.

\section{BIBLIOGRAPHIE}

ANSELMI Alessandra (2016), « Magia e stregoneria nel teatro di Nicolò Piperno, Filippo Acciajoli e Girolamo Fontana: la Noce di Benevento », P. Bertolone, A. Corea \& D. Gavrilovich (dir.), Trame di meraviglia. Studi in onore di Silvia Carandini, Rome : Universitalia.

ARETino Pietro (1969), « Dialogo nel quale la Nanna insegna a la Pippa », G. Aquilecchia (éd.), Sei giornate, Bari : Laterza, 141-355.

ARETino Pietro (2014), Teatro comico. Cortigiana (1525 e 1534) - Il Marescalco, L. D’Onghia (éd.), Parme / Milan, Guanda / Fondazione Bembo.

CAMPETELla Moreno (2014), « Les sorcières dans les comédies vénitiennes du XVI siècle, 10, 261-276.

CRUCIANI Fabrizio (1993), Teatro nel Rinascimento. Roma 1450-1550, Rome : Bulzoni.

DeL Col Andrea (2006), L'Inquisizione in Italia dal XII al XXI secolo, Milan : Mondadori, 176-193.

DHRAÏEF Beya, NeGREL Éric \& RUIMI Jennifer (dir.) (2019), Théâtre et charlatans dans l'Europe moderne, Paris : Presses Sorbonne Nouvelle.

DunI Matteo (2012), « I dubbi sulle streghe », G. Ernst (éd.), I vincoli della natura. Magia e stregoneria nel Rinascimento, Rome : Carocci, 203-221. 
ERNST Germana (1990), «I poteri delle streghe tra cause naturali e interventi diabolici. Spunti di un dibattito ", Giovan Battista Della Porta nell'Europa del suo tempo: atti del convegno Vico EquenseCastello Giusso (29 settembre-3 ottobre 1986), Naples : Guida, 167-197.

Fantini Maria Pia (2000), «Les mots secrets des prostituées (Modène, 1580-1620) », Clio. Femmes, Genre, Histoire, 11, 21-47, <https://doi.org/10.4000/clio.212>.

FERRONI Giulio (1999), Le voci dell'istrione: Pietro Aretino e la dissoluzione del teatro, Naples : Liguori.

GenTILCORE David (2006), Medical Charlatanism in Early Modern Italy, Oxford : Oxford University Press.

GianCARLi Gigio Arthemio (1991), Commedie. La Capraria - La Zingana, L. Lazzerin (éd.), Padoue : Antenore (Princeps, Mantoue : Ruffinelli, 1545).

Grazzini Anton Francesco (1976), La Strega, M. Plaisance (éd.), Abbeville : F. Paillart (Princeps, Venise : Giunti, 1582).

Groto Luigi (1599), Il Tesoro, Venise : Flli Zoppini (Princeps, Venise : F.lli Zoppini, 1583).

HIREL-WOUTS Sophie (2020), « De la trotaconventos à la buena y sabia maestra : réflexions sur la transmission des savoirs dans La Célestine », T. Rodríguez \& L. González Fernández (éds), La transmission de savoirs licites et illicites dans le monde hispanique péninsulaire (XII au XVII siècles) : hommage à André Gallego, Toulouse : Presses universitaires du Midi, 155-169.

JARDIN Jean-Pierre (2008), « Célestine et les démons », G. Martin (dir.), Fernando de Rojas, La Celestina. Comedia o tragicomedia de Calisto y Melibea, Paris : Ellipses, 81-101.

LARIVAILLE Paul (1997), Pietro Aretino, Rome : Salerno Editrice.

LETA Matteo (2019), « La riscrittura parodica del rituale magico in tre commedie del Cinquecento ", Le forme del comico: atti delle sessioni parallele del XXI Congresso dell'ADI, Firenze (6-9 settembre 2017), Florence : Soc. Ed. Fiorentina, <www.italianisti.it/pubblicazioni/atti-dicongresso/le-forme-del-comico/04_03_figorilli_leta.pdf>.

MELzI Robert C. (2000), « Celestina Italian Style », Rivista italiana di drammaturgia, 17(2), 32-43.

MiLANi Marisa (1994), Streghe e diavoli nei processi del S. Uffizio. Venezia 1554-1558, Vicence : Ghedina e Tassotti.

PADOAN Giorgio (1996), L'avventura della commedia rinascimentale, Padoue : Vallardi.

PASQUALIGO Luigi (1576), Il Fedele, Venise : B. Zaltieri.

PICo Giovan Francesco (1555), Dialogo intitolato la Strega, ouero de gli inganni de demoni, Pescia : Lorenzo Torrentino stampator ducale.

PIERI Marzia (1989), La nascita del teatro moderno in Italia tra XV e XVI secolo, Turin : Bollati Boringhieri.

PlaisAnCE Michel (2000), « Dal Candelaio di Giordano Bruno a Lo astrologo di Giovan Battista Della Porta ", S. Carandini (éd.), Teatri barocchi: tragedie, commedie pastorali nella drammaturgia europea fra ‘500 e ‘600, Rome : Bulzoni, 261-276.

PlaISANCE Michel (2005), Antonfrancesco Grazzini dit Lasca. Écrire dans la Florence des Médicis, Rome : Vecchiarelli Editore.

PORTONE Paolo (1990), Il noce di Benevento. La stregoneria e l'Italia del Sud, Milan : Xenia. 
Procaccioli Paolo (2016), « "Marginalia” aretiniani. I "secreti” della Nanna e quelli del suo autore », Bollettino d'Italianistica, 2, 46-55.

Ricchi Agostino (1998), I Tre tiranni, Gallo Anna Maria (éd.), Milan : Il Polifilo (Princeps, Venise : B. de Vitali, 1533).

SIMONATO Edoardo (2020), « Le commedie di Luigi Groto: questioni di datazione, rapporto con le fonti latine e volgari », Studi giraldiani. Letteratura e teatro, 6, 41-180.

Vescovo Pier Mario (1996), Da Ruzante a Calmo. Tra «signore comedie» e "onorandissime stampe», Padoue : Antenore.

\section{NOTES}

1. La célèbre comédie de Pietro Aretino voit le jour à Rome en 1525 ; restée manuscrite, elle est peut-être représentée pour le Carnaval ; une deuxième version remaniée est publiée à Venise en 1534 au moment où le poète et dramaturge s'y installe, dans un contexte propice également à la publication des Ragionamenti en 1534 et 1536, qui viennent compléter sa production satirique. 2. Le caractère plus ou moins fiable des dénonciations de voisins, ou de la désignation de complices de la part des suspectes de sorcellerie est justement au centre des controverses entre tenants et pourfendeurs de la chasse; voir par exemple les retranscriptions de procès dans Milani (1994). Pour une mise en perspective de ces questions et des pratiques inquisitoriales, voir Del Col (2006: 176-193).

3. Voir Melzi (2000 : 32-43). Le texte est édité à Rome en 1506, traduit par Alfonso Hordonez, familier du pape Jules II, et connaît un très vif succès dans toute la péninsule ; on en trouve deux exemplaires dans la bibliothèque du Vénitien Marin Sanudo. Les citations et renvois intertextuels émaillent les comédies de notre corpus.

4. La comédie allégorique de Ricchi (1512-1564), médecin lucquois qui a publié un traité de Galien, proche d'Aretino, a été représentée avec succès en 1530 à Bologne, pendant les fêtes pour le couronnement de Charles V ; elle est éditée à Venise (B. de Vitali) en 1533.

5. Il s'agit des trois représentants majeurs de cette congrega d'auteurs-acteurs très actifs à Venise entre 1540 et 1550 , dont les pièces souvent représentées au Carnaval articulent une riche expérimentation scénique avec les inventions de la comédie plurilingue popolaresca, se nourrissant de l'apport de bouffons et acteurs comme Burchiella, inventeur du fameux greghesco. Nous nous concentrerons sur l'œuvre de Giancarli, peintre et dramaturge au talent polymorphe proche d'Andrea Calmo (1510-1571) : La Capraria et La Zingana contaminent les sources classiques et les expérimentations plus récentes de Ruzante avec sa snaturalité, contribuant à promouvoir la comédie dialectale et plurilingue. La Zingana est publiée en 1545 à Mantoue, chez Ruffinelli, avec la protection du cardinal Ercole Gonzaga.

6. Luigi Groto (1541-1585), dit Cieco d'Adria, est un auteur de tragédies reconnu, acteur et promoteur de spectacles dans sa ville natale d'Adria. Il publie Il Tesoro à Venise (F.lli Zoppini) en 1583. Voir Simonato (2020: 41-180).

7. L'unique comédie de ce patricien et lettré vénitien (1536-1576), auteur de Lettere amorose, est éditée à Venise (Zaltieri, 1576) ; elle connaît un vif succès, avec sa veine pathétique, introspective et romanesque ; elle est adaptée en français par Pierre de Larivey en 1611.

8. Pour une étude de la production d'Antonfrancesco Grazzini (1505-1584), dans ses liens étroits avec la vie culturelle florentine et le pouvoir médicéen, et pour une analyse des différentes versions de La Strega à l'aune de la censure ecclésiastique avec laquelle il doit composer, voir Plaisance (2005). Nous citons le texte à partir de l'édition critique établie justement par Michel 
Plaisance, qui se fonde sur le manuscrit de la Bibliothèque nationale de Florence (rédaction datée autour de 1546), comparée aux éditions vénitiennes postérieures (Venise, F.lli Giunti, 1582).

9. Les abus sanglants des persécutions en Val Camonica entre 1518 et 1521 suscitent par exemple un âpre conflit entre les inquisiteurs, le podestà de Brescia et le Conseil des Dix, dont les Diarii de Marin Sanudo se font l'écho. Toujours dans la première moitié du siècle, la diffusion des théories de Pomponazzi sur les démons et les réactions indignées face à la vague de procès qui avait conduit dix condamnés au bûcher dans son petit duché poussent Gianfrancesco Pico Conte della Mirandola (1469-1533) à publier en 1523 Strix, sive de ludificatione dæemonum. Ce traité dialogué, qui vise à légitimer la chasse et les thèses du Malleus, est diffusé dans les autres États italiens en particulier grâce à la traduction en volgare par le dominicain Leandro Alberti, en 1524, avec le titre Strega o dele illusioni del demonio, puis grâce à celle de l'abbé Turino Turini en 1555.

10. Voir Portone (1990). Signalons néanmoins un corpus plus réduit et tardif d'œuvres inspirées de cet arbre légendaire où étaient censés se dérouler des sabbats endiablés. La comédie La noce maga di Benevento estirpata da S. Barbato du dramaturge napolitain Nicolo' Piperno (1666), ainsi que les intermèdes de l'opéra La caduta del Regno delle Amazzoni (1690), représentés dans des palais aristocratiques entre Naples et Rome dans le dernier tiers $d u$ XVII $^{e}$ siècle, tirent parti du thème sous un jour comique et pittoresque : les effets spectaculaires baroques se conjuguent avec une veine licencieuse inhérente au sabbat. Le père du dramaturge napolitain, Pietro Piperno, médecin de Bénévent, avait abordé la question sous un angle bien plus sérieux et érudit, dans un traité édité en latin et en italien qui a sans doute servi de source pour l'adaptation théâtrale : Della superstitiosa noce di Benevento Trattato Historico (Naples, 1640). Voir à ce sujet Anselmi (2016).

11. Vu la richesse de la bibliographie critique, nous nous bornons à quelques indications qui ne sont absolument pas exhaustives : pour un tableau d'ensemble, voir Pieri (1989) et Padoan (1996). Au sujet de l'œuvre d'Aretino, voir Ferroni (1999) et Larivaille (1997). Sur le contexte théâtral romain plus large dans lequel il s'inscrit, voir Cruciani (1993). Enfin, pour une étude d'ensemble de la comédie plurilingue d'aire vénitienne, voir Vescovo (1996).

12. Voir Plaisance (2000 : 261-276) et Leta (2019). Fait significatif, la seule étude très documentée abordant la question des sorcières dans la comédie est centrée sur l'aire culturelle vénitienne : voir Campetella (2014: 261-276).

13. Le type de la sorcière de village, plus isolée et à la marge, accusée de nuire à la communauté, provoquant sécheresses et tempêtes, n'y a guère droit de cité.

14. Dans les statuts de collèges de médecins et dans les retranscriptions des interrogatoires de procès à Rome, Modène, ou Venise, les deux épithètes strega/herbera finissent souvent par se recouper. Par ailleurs, plusieurs études ont été consacrées à l'histoire de la médecine empirique en Italie, véritable berceau du phénomène du Moyen Âge aux Lumières, en particulier à travers une remarquable exploitation des sources médicales et judiciaires : voir Gentilcore (2006).

15. Les explications font appel aux vertus hallucinogènes de certaines substances, au rôle d'un tempérament mélancolique, sans oublier les effets d'une misère à la fois matérielle et morale. Il faut noter que ces doutes, et les appels à une forme de prudence et précaution dans l'instruction des procès, se répandent au sein même de certaines instances inquisitoriales. Voir Duni (2012 : 203-221).

16. Les fèves sont mentionnées par Aloisia au sujet de Maggiorina justement dans la variante vénitienne de La Cortigiana (1534) tout comme par la Comare dans les Ragionamenti, et dans La Zingana par le valet Spingarda au sujet de Agata.

17. Voir Jardin (2008 : 81-101). Celui-ci souligne à juste titre que l'élément essentiel est l'adhésion indéniable de Célestine à son propre pouvoir magique, sa croyance en une intervention effective du démon au service de son entreprise, ce en quoi elle se distingue de la « lignée » italienne.

18. Les sorcières s'apparentent ainsi à des figures méta-théâtrales d'actrices / bonimenteuses. La mise en scène de soi, d'une parole artificieuse vantant dans des listes hyperboliques l'efficacité de remèdes empiriques, nous rapproche du thème de la charlatanerie. Les rapports entre théâtralité 
et médecine empirique dans l'Europe de l'Ancien Régime, en partie pour ce qui a trait à l'histoire de la commedia dell'arte, ont été abordés dans le volume collectif Théâtre et charlatans dans l'Europe moderne (2019). Rappelons aussi que lors de la grande croisade contre le théâtre à l'âge baroque, les théologiens qualifient souvent les nouvelles actrices de métier de sorcières ou de magiciennes.

19. Dans l'édition de sa Magia naturalis de 1558, Della Porta explique le vol au sabbat des sorcières comme une expérience onirique hallucinatoire, et décrit avec minutie la composition d'un des fameux onguents. Voir Ernst (1990: 167-197).

20. Pour la "questione femminile» dans cette comédie, voir les remarques de Simonato (2020 : 169).

21. Voir Hirel-Wouts (2020 : 155-169). Passant en revue les différents savoirs dont cette figure tutélaire est à la fois détentrice et passeuse, l'étude montre «à quel point la transmission est organiquement liée au personnage de Célestine, non pas en tant que sorcière ou entremetteuse, mais en tant que détentrice d'une connaissance fondamentale concernant l'être humain, connaissance par nature inscrite en marge des savoirs licites habituellement réservés à l'homme » (ibid. : 156).

22. Voir Fantini (2000: 21-47).

23. Voir Aretino (1969: 141-355).

24. L'objet-livre est un accessoire prisé dans les rituels de la fausse magie au masculin, dans Il Tesoro par exemple, associé aux disciplines plus nobles, magie naturelle, alchimie, ou astrologie, même parodiée. Le grimoire est évincé de la sphère opérationnelle des sorcières; le rapport à l'écrit se joue par exemple dans les petits papiers secrets sur lesquels sont inscrits les incantations et les noms des esprits dans le sortilège de Medusa au cimetière.

25. Voir Procaccioli (2016: 46-55).

26. Nous citons à partir de la deuxième traduction en italien de l'abbé Turino Turini : Pico (1555 : 76-77).

27. Voir l'introduction de L. Lazzerin à La Zingana dans Giancarli (1991 : XII-XIII) : «Il microcosmo della commedia [...] è ormai metafora della complessità del reale, della molteplicità dei punti di vista: specchio di un'epoca che aveva smarrito la fiducia in un significato oggettivo, immutabile della realtà, $e$ intuito la labilità del confine fra essere e parere, esperienza e illusione. »

\section{RÉSUMÉS}

Dans la comédie de la Renaissance italienne, depuis La Cortigiana de 1525 de Pietro Aretino jusqu'à la comédie polyglotte vénitienne de la deuxième moitié du siècle, s'affirme progressivement un type comique et pittoresque aux attributs stéréotypés, la vieille entremetteuse/sorcière, dans la lignée de La Célestine de Fernando de Rojas, reflet du phénomène diffus des "stregherie/herberie » en milieu urbain. Notre article se propose d'analyser les ambivalences et paradoxes qui soustendent la représentation : figure grotesque et négative du désordre moral et de la charlatanerie, la sorcière peut se charger de valeurs positives, dépositaire d'un savoir empirique utile à la communauté, emblème d'une condition féminine autonome par rapport à l'autorité masculine. Le jeu de relativisation ironique des discours et la polyphonie théatrale permettent ainsi de questionner l'imaginaire social et la catégorie même de «strega " promue par inquisiteurs et démonologues. 
In Italian Renaissance comedy, from Pietro Aretino's Cortigiana of 1525 to the Venetian polyglot comedy of the second half of the century, a comic and picturesque character with stereotyped attributes, the old matchmaker/witch, in the line of Fernando de Rojas' La Celestina, progressively asserts itself, reflecting the diffuse phenomenon of the "stregherie/herberie" in urban environments. Our article sets out to analyze the ambivalences and paradoxes that underlie its portrayal: though often rendered as grotesque and negative figures of moral disorder and charlatanism, witches can also be charged with positive values, as the depositaries of empirical knowledge useful to the community and as emblems of a female condition independent from male authority; playing with ironic relativization of discourse and theatrical polyphony both bring this social imaginary into question, along with the very category of "strega" as promoted by inquisitors and demonologists.

INDEX

Mots-clés : comédie de la Renaissance italienne, personnage de l'entremetteuse/sorcière, savoirs empiriques au XVIe siècle, théâtre et magie au XVIe siècle

Keywords : Italian Renaissance comedy, character of the witch/matchmaker, empirical knowledge in the 16th century, theater and magic in the 16th century

\section{AUTEUR}

COSTANZA JORI

Sorbonne Nouvelle

costanzajori@hotmail.com 